\title{
Evidence-Based Nursing: past, present, and future
}

\begin{abstract}
With this issue, we begin the third volume of Evidence-Based Nursing $(E B N)$. In this editorial, we describe how the journal is produced, identify the journals from which we abstract the most studies and share the results of a recent subscriber survey. We then update you on new and future developments in the production of $E B N$.
\end{abstract}

\section{Steps in creating $E B N$}

$E B N$ shares a similar production process to sibling abstract journals, Evidence-Based Medicine, ${ }^{1}$ Evidence-Based Mental Health, and ACP Journal Club. It begins with the meticulous scrutiny of every issue of approximately 140 clinical journals (a complete list appears in each issue of $E B N$ ) by research associates, who have extensive research training and experience in information science and clinical epidemiology and biostatistics. After identifying every primary study and systematic review related to prevention or treatment, assessment (screening or diagnosis), prognosis, causation, quality assurance, economics of healthcare programmes or interventions, clinical prediction guides, and qualitative studies, they apply appropriate methodological criteria outlined in the Purpose and Procedure section of each issue. In the next step, each article that "passes" the appraisal is reviewed by the 3 editors to identify those of most relevance to nursing practice. Because we do not have expertise in all areas of nursing, colleagues from around the world help us to determine the relevance of some of the articles. The third step involves the preparation of a structured abstract that summarises the question(s), design, setting, patients, results, and evidence-based conclusions for each of the articles that have been selected for inclusion. In step 4 , the associate editors collaborate with the research associate in editing each abstract. We then invite a nurse with expertise in the topic area to write a commentary that discusses whether and how the study results might be implemented in nursing practice. In step 5, the associate editor, through exchanges with the commentator, fine tunes the abstract and commentary. Step 6 brings in the lead author of the paper who reviews the abstract and commentary for accuracy. Before submitting to the publishers, the abstract and commentary are reviewed once more by a research associate, who has not read the original paper, to assess their clarity. The material is then sent to the technical editor in the UK who works closely with the research associates through 3 cycles of typesetting and proofreading before the issue is published. This multistep process relies on the collaboration of numerous individuals from around the world and is crucial to maximise the accuracy and usefulness of the abstract and commentary.

\section{Identification of studies for inclusion in EBN}

The 192 abstracts that appeared in our first 2 volumes were selected from 67 of about 140 journals that are reviewed regularly. The top 10 journals (table) yielded $97(51 \%)$ of the abstracted articles. Only 2 of the top 10 journals are specifically published for nurses, Journal of Advanced Nursing and Cancer Nursing. The remainder are general healthcare journals, specialty healthcare journals, a journal focusing on qualitative research, and the Cochrane Library. These data tell us 3 things: firstly, many good quality research articles are published that are of relevance to nursing practice; secondly, many nurses submit their research papers to non-nursing journals; and, thirdly, many research studies published in general healthcare journals written by non-nurses are relevant to nursing practice. Few nurses would be able to regularly read these 10 journals as well as the 57 journals that yielded the remaining $49 \%$ of the abstracted articles, emphasising the need for a journal like $E B N$.

As well as the articles that are chosen by the editors for abstraction, many articles pass all the methodological criteria but are not abstracted because, in the judgment of the editors, their findings are less applicable to nursing practice. Over the past 2 years, we have identified at least 400 such articles. To make these studies available to our readers, we include a selected list in "other articles noted" in the last issue (October) of each volume. The table also summarises the number of articles contributed by the top 10 journals to this list.

\section{Subscriber survey}

The high subscription rate to $E B N$ is a strong indication of nurses' interest in evidence-based practice. As of October 1999, we had over 8000 subscribers worldwide. In January 1999, the publishers sent a questionnaire to a random sample of 3785 current and lapsed subscribers requesting opinions about $E B N$. We thank all the readers who responded for their valuable input.

Most respondents were women, aged 35-44 years, who qualified as registered nurses 16-20 years ago, and identified clinical practice as the primary focus of their job. Most current subscribers rated the journal as "excellent" or "good" and would like to see the journal published more frequently. Some respondents indicated that the understandability of the abstracts and editorials could be improved. Some also noted that only a few abstracts in any one issue were of relevance to their specific areas of practice. Because $E B N$ is intended to keep nurses from any specialty area up to date on current research developments and because we review such a breadth of healthcare journals, the abstract topics are diverse. We outline a pilot project to address this issue in the discussion of future developments.

\section{Journals contributing the most articles to EBN (1998-9)}

\begin{tabular}{llll} 
Journal & $\begin{array}{l}\text { Number of } \\
\text { articles } \\
\text { abstracted }\end{array}$ & $\begin{array}{l}\text { Number of } \\
\text { articles cited in } \\
\text { "Other articles } \\
\text { noted" }\end{array}$ & Total \\
\hline JAMA & 15 & 25 & 40 \\
\hline BMJ & 14 & 13 & 27 \\
\hline J Adv Nurs & 13 & 19 & 32 \\
\hline Cochrane Library & 10 & 26 & 36 \\
\hline Pediatrics & 9 & 15 & 24 \\
\hline N Engl J Med & 9 & 12 & 21 \\
\hline Qual Health Res & 9 & 12 & 21 \\
\hline Arch Intern Med & 6 & 10 & 16 \\
\hline Am J Obstet Gynecol & 6 & 5 & 11 \\
\hline Cancer Nurs & 6 & 3 & 9 \\
\hline
\end{tabular}




\section{What's new in the third volume of EBN?}

You may have already noticed a new look to the cover of this issue. We have been fortunate to have Dr Caroline Brown, a nurse, perinatal consultant, and assistant professor at West Virginia University School of Nursing in Morgantown, West Virginia, USA, serve as the photographer for our new cover. Dr Brown has won 22 photographic prizes and exhibited her photographs in various museums in North America. On the cover, Dr Brown has captured many of the dimensions of nursing.

You will notice a few new names on the inside cover. We are pleased to have recruited 5 associate editors: Caroline Carlisle, at the University of Liverpool, UK; Marlene Z Cohen, at the University of Texas and University of Texas MD Anderson Cancer Center, USA; Rita Schreiber, at the University of Victoria, Canada; Carl Thompson, at the University of York, UK; and Sally Thorne, at the University of British Columbia, Canada. These colleagues have reviewed articles for possible inclusion in the journal and have served as associate editors on several abstracts. We welcome them on board and are excited about working with them. Also, we have recruited Dr Geoff Norman, professor in the Department of Clinical Epidemiology and Biostatistics at McMaster University and author of numerous books on biostatistics and epidemiology, to help us to sort out thorny statistical issues that arise.

You will notice that we have incorporated colour into the journal and changed the format of the abstract pages. The research question is now more obvious through the use of a larger font and a change in format; the commentary now appears in a blue box separating it more clearly from the abstract

Under the "design" section of each of the quantitative abstracts, we now include information about allocation concealment and blinding (masking). These concepts will be the subject of a future EBN Notebook and therefore we will explain them only briefly here. The most important feature of a randomised controlled trial is the randomisation of participants to intervention or control groups. Randomisation is important because it removes the possibility of bias in group allocation and ensures the even distribution between groups of known and unknown factors that affect the outcomes of interest. Thus, the groups are similar in every way except exposure to the intervention, and differences in outcome between the groups can be confidently regarded as resulting from the intervention. To maximise the similarities among groups, the process of allocating participants to groups should be both random and unpredictable (concealed). An example of an acceptable randomisation strategy is a computer generated random number table, whereas strategies such as allocating every second patient to the intervention group are quasi-random, predictable, and, open to manipulation. Once the randomisation sequence has been established, the second process involves the concealment of that sequence. Concealment of allocation protects those in a trial from knowing upcoming assignments in advance and consciously or unconsciously manipulating the allocation schedule. An example of concealed allocation is telephoning a central registry to be told which group to assign the patient to; an example of unconcealed allocation is posting a list of the randomisation sequence on the bulletin board of the recruitment site or the allocation of alternate patients. Those responsible for enrolling patients could easily see which group the next patient will be allocated to and then could manipulate the order in which patients are seen so that those with better prognoses are assigned to the intervention group and those with poorer prognoses to the control group, or vice versa. ${ }^{2}$

$E B N$ abstracts now also describe whether the studies were blinded and who was blinded. Blinding refers to whether the study personnel and participants knew the groups to which participants were assigned; blinding reduces various kinds of bias.

\section{Future developments}

The $E B N$ web site can be found at http://www.evidencebased nursing.com. We are working to expand this web site and hope to have a full text web site available free to all subscribers by June 2000. This will include search facilities; links to an interactive glossary; links to primary papers if they are available online; full purpose and procedure information; and letters and debate.

The educational preparation of many nurses has not included critical appraisal. As a result, although nurses are highly motivated to incorporate evidence into their practice, they may feel disadvantaged by their lack of training in this area. In an effort to address this, we have included the EBN Notebook, in which we are reviewing steps in the research and critical appraisal process, and a glossary. We are considering supplementing these by choosing one study per issue to which we would devote 2 pages rather than 1 page. The abstract and commentary of this study would be complemented by a detailed, clear description of methodological concepts arising from the paper.

To supplement $E B N$, which includes abstracts on a broad range of current topics, we are exploring the feasibility of publishing an additional specialty specific issue each year. For example, we have been surprised at the number of scientifically sound studies of relevance to neonatal nurses. Yet, we cannot publish abstracts for all of these studies without seriously compromising the journal's relevance to nurses who are not neonatal nurses. The specialty specific issues of $E B N$ would bring together all of the recently published studies in given topic areas, such as neonatal nursing, care of the elderly, and community health.

Editing the journal has given us an opportunity to learn that nurses are highly motivated to become evidence-based practitoners, that many high quality studies of relevance to nursing are published in various healthcare journals, and that reviewing the many journals that publish relevant studies without the help of an abstraction journal would be a daunting task.

We take this opportunity to thank the many people that make this journal possible: the associate editors who help to identify articles to abstract and who edit abstracts and commentaries, the commentators, those who have written or peer reviewed editorials, the staff in the Health Information Research Unit at McMaster University, the staff at the BMJ Publishing Group and the RCN Publishing Company, and our coordinating editor, Brian Haynes.

\section{ALBA DiCENSO, RN, PhD NICKY CULLUM, RN, PhD DONNA CILISKA, RN, PhD Editors, Evidence-Based Nursing SUSAN MARKS, BA, BEd Research Associate, Evidence-Based Nursing}

1 Sackett DL, Haynes RB. 13 steps, 100 people, and 1000000 thanks [EBM notebook]. Evidence-Based Medicine 1997;July-Aug;2:101-2.

2 Schulz KF. Assessing allocation concealment and blinding in randomised controlled trials: why bother? [editorial] Evidence-Based Mental Health, in press. 